\title{
Linear switched DAEs: Lyapunov exponents, a converse Lyapunov Theorem, and Barabanov norms
}

\author{
Stephan Trenn and Fabian Wirth
}

\begin{abstract}
For linear switched differential algebraic equations (DAEs) we consider the problem of characterizing the maximal exponential growth rate of solutions. It is shown that a finite exponential growth rate exists if and only if the set of consistency projectors associated to the family of DAEs is product bounded. This result may be used to derive a converse Lyapunov theorem for switched DAEs. Under the assumption of irreducibility we show that a construction reminiscent of the construction of Barabanov norms is feasible as well.
\end{abstract}

\section{INTRODUCTION}

In this paper it is our aim to establish a converse Lyapunov theorem and to construct a Barabanov norm for switched DAEs of the form

$$
E_{\sigma(t)} \dot{x}(t)=A_{\sigma(t)} x(t), x\left(t_{0}-\right)=x_{0} \in \mathbb{R}^{n}
$$

where the switching signals $\sigma$ satisfy, for some finite or infinite index set $\mathcal{I}$,

$$
\sigma \in \Sigma:=\left\{\begin{array}{l|l}
\sigma: \mathbb{R} \rightarrow \mathcal{I} & \begin{array}{l}
\sigma \text { is piecewise constant with } \\
\text { locally finitely many switches }
\end{array}
\end{array}\right\} .
$$

Switched DAEs (1) have attracted some interest in recent years [17]. A general solution theory has recently been developed in [15], [16]. First results on the stability of switched DAEs have been derived in [9], [11], [10]. In particular, it follows from the general theory that for arbitrary switched linear DAEs solutions need to be defined in the space of piecewise smooth distributions, which potentially include Dirac impulses. The problem in DAEs is the discontinuity due to the restriction of the state to the consistency space, see e.g. [13]. As an impulse in a solution prevents a reasonable notion of stability, we only consider the situation where the solutions of (1) do not contain Dirac impulses. As we consider arbitrary initial values, non-existence of Dirac impulses in the solution is equivalent to the index-one assumption of the matrix pairs $\left(E_{i}, A_{i}\right)$.

For switched linear ordinary differential inclusions converse Lyapunov theorems were obtained in [1], [19] and we adapt some of the ideas to the setting considered here. The class of systems we consider here can be modeled within the class of hybrid systems in the framework discussed in [6]. In particular, in this general context converse Lyapunov theorems are available, [3]. These are also applicable in

This work was supported by the DFG grant Wi1458/10-1.

$\mathrm{S}$. Trenn is with the Department of Mathematics, University of Kaiserslautern, 67663 Kaiserslautern, Germany

email: trenn@mathematik. uni-kl. de

F. Wirth is with the Institute for Mathematics, University of Würzburg, 97074 Würzburg, Germany

email: wirth@mathematik.uni-wuerzburg.de principal for the systems studied here, but our construction provides some more information as the exponential growth rate of the system is characterized precisely. This has proven to be an essential tool for switched linear ordinary differential equations and we expect the same to be true for switched DAEs (1).

The paper is organized as follows. In Section II we show that a switched DAE under certain conditions can be rewritten as a switched ODE with jumps. We study the latter in its own right in Section III and derive a converse Lyapunov theorem (Corollary 14) as well as conditions for the existence of a Barabanov norm (Theorem 16). These results can then be formulated for switched DAEs which is done in the concluding Section IV.

Throughout this paper $\|\cdot\|$ denotes an arbitrary (but fixed) norm on $\mathbb{R}^{n}$ as well as the corresponding induced matrix norm.

\section{SWITCHED DAES AND SWITCHED ODES WITH JUMPS}

In this section we will show that under certain assumptions on (1) we can reformulate (1) for a fixed switching signal $\sigma \in \Sigma$ as a switched ODE with jumps, given by

$$
\begin{aligned}
\dot{x}(t) & =A_{i_{k}}^{\mathrm{diff}} x(t), \text { on }\left(t_{k}, t_{k+1}\right) \\
x\left(t_{k}+\right) & =\Pi_{i_{k}} x\left(t_{k}-\right), \quad x\left(t_{0}-\right)=x_{0} \in \mathbb{R}^{n}
\end{aligned}
$$

where $t_{k}, k \in \mathbb{N}$ are the ordered switching times of $\sigma$ beginning at $t_{0} \in \mathbb{R}$ and $i_{k} \in \mathcal{I}$ is such that $\sigma(t)=i_{k}$ on $\left(t_{k}, t_{k+1}\right)$, furthermore, $\left(A_{i}^{\text {diff }}, \Pi_{i}\right) \in \mathcal{A} \Pi$ for all $i \in \mathcal{I}$, where

$$
\mathcal{A} \Pi=\left\{(A, \Pi) \in\left(\mathbb{R}^{n \times n}\right)^{2} \mid \Pi^{2}=\Pi, A \Pi=\Pi A\right\} .
$$

Here $A_{i}^{\text {diff }}$ characterizes the differential dynamics of the DAE $E_{i} \dot{x}=A_{i} x$ and $\Pi_{i}$ is the so called consistency projector.

For deriving the connection between (1) and (2) we start by collecting some facts about non-switched DAEs

$$
E \dot{x}=A x .
$$

Definition 1 (Regularity): A matrix pair $(E, A) \in$ $\mathbb{R}^{n \times n} \times \mathbb{R}^{n \times n}$ (and the associated DAE) is called regular if, and only if, the polynomial $\operatorname{det}(s E-A)$ is not the zero polynomial.

An important characterization of regularity is the quasiWeierstrass form:

Lemma 2 (Quasi-Weierstrass form, [18], cf. [5], [2]):

The matrix pair $(E, A)$ is regular if, and only if, there exist invertible $S$ and $T$ such that

$$
(S E T, S A T)=\left(\left[\begin{array}{cc}
I & 0 \\
0 & N
\end{array}\right],\left[\begin{array}{ll}
J & 0 \\
0 & I
\end{array}\right]\right),
$$


where $N \in \mathbb{R}^{n_{2} \times n_{2}}$ is nilpotent and $J \in \mathbb{R}^{n_{1} \times n_{1}}, n_{1}+n_{2}=$ $n$. The smallest number $\nu \in \mathbb{N}$ such that $N^{\nu}=0$ is called the index of the regular pair $(E, A)$.

Following [2] we call (4) a quasi-Weierstrass form, as we do not assume that $N$ and $J$ are in Jordan canonical form. Also, [2] describes a convenient way to obtain a quasiWeierstrass form (and the corresponding transformation matrices) utilizing the Wong sequences.

We are now able to characterize the (classical) solutions of the DAE (3). Here classical means continuously differentiable or absolutely continuous.

Lemma 3 (Differential flow matrix, [14]): Consider a regular matrix pair $(E, A)$ with transformation matrices $S$ and $T$ which put $(E, A)$ in a quasi-Weierstrass form. Let the differential flow matrix of the DAE (3) be given by

$$
A^{\text {diff }}:=T\left[\begin{array}{cc}
I_{n_{1}} & 0 \\
0 & 0
\end{array}\right] S A=T\left[\begin{array}{ll}
J & 0 \\
0 & 0
\end{array}\right] T^{-1},
$$

then $x$ solves the DAE (3) if, and only if, $x$ solves

$$
\dot{x}=A^{\text {diff }} x, \quad x(0) \in \mathcal{V}^{*}:=\operatorname{im} T\left[\begin{array}{cc}
I_{n_{1}} & 0 \\
0 & 0
\end{array}\right] .
$$

In particular, the solutions evolve within the consistency space $\mathcal{V}^{*}$ which is in general not the whole $\mathbb{R}^{n}$. This is important when studying switched DAEs (1) as the consistency spaces corresponding to different modes need not coincide. In particular, existence of classical solution cannot be expected in general. In order to continue a solution from a switching instant with an appropriate jump we have to utilize the solution space of piecewise-smooth distributions as introduced in [15], [16]. In this distributional framework the jumping behavior at a switching instant follows from studying the solution of the initial trajectory problem (ITP) for the DAE (3):

$$
\begin{aligned}
x_{(-\infty, 0)} & =x_{(-\infty, 0)}^{0} \\
(E \dot{x})_{[0, \infty)} & =(A x)_{[0, \infty)}
\end{aligned}
$$

where $x^{0}$ is an arbitrary past trajectory; in particular, it is not assumed that $x^{0}$ evolves within $\mathcal{V}^{*}$. The following results now establishes existence and uniqueness of solutions of the ITP in the piecewise-smoothly distributional framework.

Lemma 4 (Consistency projector, [15]): The ITP (5) has a unique (distributional) solution for all initial trajectories $x^{0}$ if, and only if, $(E, A)$ is regular. In particular, each local solution of the switched DAE (1) can uniquely be extended forward in time up to infinity. Furthermore, the jump in the ITP (5) is given by the consistency projector

$$
x(0+)=\Pi x^{0}(0-) \text { where } \Pi=T\left[\begin{array}{cc}
I_{n_{1}} & 0 \\
0 & 0
\end{array}\right] T^{-1},
$$

and where $T \in \mathbb{R}^{n \times n}$ is as in Lemma 2 .

However, this unique solution might exhibit "infinite peaks" in the form of the Dirac impulse (and its derivatives). In the context of stability the existence of infinite peaks is clearly an undesirable behavior. It is well known (cf. [17]), that the ITP (5) does not produce Dirac impulses for arbitrary initial trajectories if, and only if, $N=0$ in the quasiWeierstrass form (4), i.e. the matrix pair $(E, A)$ has index one.
Theorem 5 (Connection between (1) and (2)): Consider the switched DAE (1) with regular matrix pairs $\left(E_{i}, A_{i}\right)$, $i \in \mathcal{I}$, of index one and switching signal $\sigma \in \Sigma$. Let

$$
\mathcal{M}^{\text {jump }}:=\left\{\left(A_{i}^{\text {diff }}, \Pi_{i}\right) \mid i \in \mathcal{I}\right\},
$$

where $A_{i}^{\text {diff }}, \Pi_{i}$ are the flow matrix and consistency projector of $\left(E_{i}, A_{i}\right)$ as in Lemmas 3 and 4 . Then

$$
\mathcal{M}^{\text {jump }} \subseteq \mathcal{A} \Pi
$$

and $x$ is a solution on $[0, \infty)$ of the switched DAE (1) with initial condition $x(0-)=x_{0}$ if, and only if, $x$ is a solution on $[0, \infty)$ of the switched ODE with jumps (2) corresponding to $\mathcal{M}^{\text {jump }}$ and with initial condition $x(0-)=x_{0}$.

Proof: First note that commutativity of $A^{\text {diff }}$ and $\Pi$ corresponding to a regular matrix pair $(E, A)$ easily follows from the definition. From Lemma 3, Lemma 4 and impulsefreeness due to index one we can conclude that the solution $x$ of the switched DAE (1) with switching signal $\sigma \in \Sigma$ and initial condition $x(0-)=x_{0}$ is given by

$$
\begin{array}{r}
x(t)=e^{A_{\sigma\left(t_{k}+\right)}^{\text {diff }}\left(t-t_{k}\right)} \Pi_{\sigma\left(t_{k}+\right)} e^{A_{\sigma\left(t_{k-1}+\right)}^{\text {diff }}\left(t_{k}-t_{k-1}\right)} \Pi_{\sigma\left(t_{k-1}+\right)} \\
\cdots e^{A_{\sigma\left(t_{0}+\right)}^{\text {diff }}\left(t_{1}-t_{0}\right)} \Pi_{\sigma\left(t_{0}+\right)} x_{0}
\end{array}
$$

where $t \in\left[t_{k}, t_{k+1}\right)$ and $0=t_{0}<t_{1}<t_{2}<\ldots$, are the switching times of $\sigma$. This already shows the claim of the theorem.

\section{A CONVERSE LYAPUNOV THEOREM AND}

\section{BARABANOV NORMS FOR SWITCHED ODES WITH JUMPS}

In this section we consider switched ODEs with jumps in its own right because a converse Lyapunov Theorem and a Barabanov norm construction is itself of interest for switched ODEs with jumps. To this end, let for some finite or infinite index set $\mathcal{I}$

$$
\mathcal{M}^{\text {jump }}:=\left\{\left(A_{i}, \Pi_{i}\right) \in\left(\mathbb{R}^{n \times n}\right)^{2} \mid i \in \mathcal{I}\right\} \subseteq \mathcal{A} \Pi
$$

be a compact set. For each $\sigma \in \Sigma$ we associate to $\mathcal{M}^{\text {jump }}$ a switched ODE with jumps

$$
\begin{aligned}
\dot{x}(t) & =A_{\sigma(t)} x(t), \text { on }\left(t_{k}, t_{k+1}\right) \\
x\left(t_{k}+\right) & =\Pi_{\sigma\left(t_{k}+\right)} x\left(t_{k}-\right), \quad k=0,1,2, \ldots, \\
x\left(t_{0}-\right) & =x_{0} \in \mathbb{R}^{n},
\end{aligned}
$$

where $t_{0}<t_{1}<\ldots<t_{k}<t_{k+1}<\ldots, k \in \mathbb{N}$, are the switching times of $\sigma$ (beginning at $t_{0}$ ). Note that, although we do not assume that $(A, \Pi) \in \mathcal{M}^{\text {jump }}$ are induced by some regular matrix pair, we do assume commutativity of $A$ and $\Pi$ for all $(A, \Pi) \in \mathcal{M}^{\text {jump }}$ which will play an important role later (see Lemma 6 and Remark 7).

\section{A. Growth rates for switched ODEs with jumps}

The solution of the switched ODE (7) is, for $t \in\left[t_{k}, t_{k+1}\right)$, given by a formula analogous to (6).

Hence we can define the evolution operator $\Phi^{\sigma}\left(\cdot, t_{0}\right)$ : $\left(t_{0}, \infty\right) \rightarrow \mathbb{R}^{n \times n}$ corresponding to a fixed switching signal $\sigma$ such that for all solution $x$ and all $t>t_{0}$

$$
x(t)=\Phi^{\sigma}\left(t, t_{0}\right) x\left(t_{0}-\right) .
$$


Since the considered set of switching signals is shift invariant, the set of all evolution operations only depends on the time $\operatorname{span} t>0$, i.e.

$$
\begin{aligned}
\mathcal{S}_{t}:=\left\{\Phi^{\sigma}\left(t_{0}+t, t_{0}\right) \mid t_{0} \in \mathbb{R}, \sigma \in \Sigma\right\} \\
=\left\{\begin{array}{l|l}
\prod_{j=1}^{k} e^{A_{j} \tau_{j}} \Pi_{j} \mid \begin{array}{l}
k \in \mathbb{N},\left(A_{j}, \Pi_{j}\right) \in \mathcal{M}^{\text {jump }}, \tau_{1}>0, \\
\ldots, \tau_{k-1}>0, \tau_{k} \geq 0, \sum_{j=1}^{k} \tau_{j}=t
\end{array}
\end{array}\right\}
\end{aligned}
$$

The set $\mathcal{S}_{t}$ defines all possible evolutions of length $t$ that can be realized by the switched ODE with jumps defined by $\mathcal{M}^{\text {jump }}$ and (7) if we admit arbitrary $\sigma \in \Sigma$. Note that the definition assumes that there is at most one jump at each time instant, i.e. $x(t+)=\Pi_{p} \Pi_{q} x(t-)$ is not possible for $p, q \in \mathcal{I}$ with $p \neq q$. However, these "multiple" jumps are in the closure of $\mathcal{S}_{t}$ which will play some role later.

We highlight the important property that

$$
\mathcal{S}:=\bigcup_{t \geq 0} \mathcal{S}_{t}
$$

is a semigroup with the following factorization property.

Lemma 6 (Semigroup): For all $s, t>0$ it holds that

$$
\begin{aligned}
\mathcal{S}_{s+t}=\mathcal{S}_{s} \mathcal{S}_{t} & := \\
& \left\{\Phi_{s} \Phi_{t} \in \mathbb{R}^{n \times n} \mid \Phi_{s} \in \mathcal{S}_{s}, \Phi_{t} \in \mathcal{S}_{t}\right\} .
\end{aligned}
$$

Proof: It is clear by (8), that $\mathcal{S}_{s} \mathcal{S}_{t} \subseteq \mathcal{S}_{s+t}$. Conversely, let $\Phi \in \mathcal{S}_{s+t}$, i.e. $\Phi$ is the flow at $s+t$ of the switched ODE (7) for a fixed switching signal. If $t$ is a switching instant of $\sigma$ there is nothing to show. Otherwise there are two consecutive switches $t_{k}, t_{k+1}$ with $t_{k}<t<t_{k+1}$. First observe that commutativity of $\Pi_{k}$ and $A_{k}$ implies commutativity of $\Pi_{k}$ and $e^{A_{k} \tau}$ for all $\tau>0$. Secondly, $\Pi_{k}^{2}=\Pi_{k}$, hence

$$
e^{A_{k}\left(t_{k+1}-t_{k}\right)} \Pi_{k}=e^{A_{k}\left(t_{k+1}-t\right)} \Pi_{k} e^{A_{k}\left(t-t_{k}\right)} \Pi_{k} .
$$

This may be used to represent $\Phi$ as the product of elements in $\mathcal{S}_{s}$ and $\mathcal{S}_{t}$ by introducing an artificial switching instant at time $t$.

Remark 7 (Commutativity condition): To show the semigroup property (10) the commutativity condition $A \Pi=\Pi A$ for all $(A, \Pi) \in \mathcal{M}^{\text {jump }}$ is essential. However, if also ODEs (7) with arbitrary jumps should be considered, one could add the artificial ODE corresponding to $(A, I)$, i.e. an ODE without a jump, to recover the semigroup property. It is then possible to introduce "artificial" switches anywhere without altering the solution. If, however, a jump must occur whenever the systems jumps from one mode to a different mode then this approach does not work because the semigroup also contains solutions corresponding to switches without jumps.

We now define the exponential growth rate of (7).

Definition 8 (Exponential growth rate): For $t>0$ define first the exponential growth bound of (7) at time $t$ by

$$
\lambda_{t}\left(\mathcal{S}_{t}\right):=\sup _{\Phi_{t} \in \mathcal{S}_{t}} \frac{\ln \left\|\Phi_{t}\right\|}{t} \in \mathbb{R} \cup\{-\infty,+\infty\},
$$

The exponential growth rate, or upper Lyapunov exponent, of the semigroup $\mathcal{S}$ is then given by

$$
\lambda(\mathcal{S}):=\lim _{t \rightarrow \infty} \lambda_{t}\left(\mathcal{S}_{t}\right)
$$

provided the limit exists and is finite.

Note that we did not exclude $\lambda_{t}\left(\mathcal{S}_{t}\right)= \pm \infty$ at this point. Still, for every solution $x$ of (7),

$$
\|x(t)\|=\left\|\Phi_{t} x_{0}\right\| \leq\left\|\Phi_{t}\right\|\left\|x_{0}\right\| \leq e^{\lambda_{t}\left(\mathcal{S}_{t}\right) t}\left\|x_{0}\right\|
$$

which motivates the term "exponential growth bound". For switched ODEs without jumps, i.e.

$$
\forall(A, \Pi) \in \mathcal{M}^{\text {jump }}: \quad \Pi=I
$$

and compact $\mathcal{M}^{\text {jump }}$ it can be shown [7] that the associated evolution operators $\mathcal{S}_{t}$ are bounded and, furthermore, the growth can be bounded exponentially, i.e. there exist $\lambda>0$ such that for all $t>0$ and $\Phi_{t} \in \mathcal{S}_{t}$

$$
\left\|\Phi_{t}\right\| \leq e^{\lambda t}
$$

or, equivalently, for all solutions $x$ of the switched ODE

$$
\|x(t)\| \leq e^{\lambda t}\left\|x_{0}\right\|
$$

In particular, $\lambda(\mathcal{S})$ exists and is finite for switched ODEs without jumps and with a compact $A$-matrices set.

Surprisingly, this property does not hold anymore when considering switched ODEs with jumps; the sets $\mathcal{S}_{t}$ can actually be unbounded for finite $t$. See the following example.

Example 9 (Unbounded evolution operators): Let $\mathcal{M}^{\text {jump }}=\left\{\left(0, \Pi_{1}\right),\left(0, \Pi_{2}\right)\right\}$ with projectors

$$
\Pi_{1}=\left[\begin{array}{ll}
0 & 1 \\
0 & 1
\end{array}\right], \quad \Pi_{2}=\left[\begin{array}{ll}
0 & 0 \\
1 & 1
\end{array}\right]
$$

Note that $\mathcal{S}_{t}$ contains all possible finite products of $\Pi_{1}$ and $\Pi_{2}$. It is easily seen that

$$
\left(\Pi_{1} \Pi_{2}\right)^{k}=\left[\begin{array}{ll}
1 & 1 \\
1 & 1
\end{array}\right]^{k}=2^{k-1} \Pi_{1} \Pi_{2}
$$

hence $\mathcal{S}_{t}$ is unbounded for each $t>0$ because in the setup considered here there is no upper bound on the number of switches in an interval $[0, t]$.

The previous example showed that the set of the jump maps

$$
\mathcal{M}_{\Pi}^{\text {jump }}:=\left\{\Pi \in \mathbb{R}^{n \times n} \mid \exists A:(A, \Pi) \in \mathcal{M}^{\text {jump }}\right\}
$$

plays a vital role. Recall that a nonempty set of matrices $\mathcal{B} \subseteq \mathbb{R}^{n \times n}$ is called product bounded [4], if the set of all possible products

$$
\mathcal{S}_{\mathbb{N}}(\mathcal{B}):=\left\{B_{k} B_{k-1} \ldots B_{1} \mid k \in \mathbb{N}, B_{j} \in \mathcal{B}\right\}
$$

is bounded. By a well known result, [8, Theorem 3], $\mathcal{B}$ is product bounded, if and only if there exists a norm $\|\cdot\|$ on $\mathbb{R}^{n}$ such that for the associated operator norm we have

$$
\|B\| \leq 1, \quad \text { for all } B \in \mathcal{B} .
$$

The following result gives a characterization of boundedness of the sets $\mathcal{S}_{t}$.

Proposition 10 (Boundedness of $\mathcal{S}_{t}$ ): Assume that the set $\mathcal{M}_{A}^{\text {jump }}:=\left\{A \in \mathbb{R}^{n \times n} \mid \exists \Pi:(A, \Pi) \in \mathcal{M}^{\text {jump }}\right\}$ is bounded. Then the following statements are equivalent:

(i) $\mathcal{M}_{\Pi}^{\text {jump }}$ is product bounded. 
(ii) there exists a $t>0$ such that $\mathcal{S}_{t}$ is bounded.

(iii) for all $t>0$ the set $\mathcal{S}_{t}$ is bounded.

Of course, the bound in (iii) may depend on $t$.

Proof: It is clear that (iii) implies (ii). To prove (i) $\Rightarrow$ (iii), assume that $\mathcal{M}_{\Pi}$ is product bounded. In this case we may choose a norm $\|\cdot\|$ on $\mathbb{R}^{n}$ so that $\|\Pi\| \leq 1$ for all $\Pi \in \mathcal{M}_{\Pi}^{\text {jump }}$. Now corresponding to the norm $\|\cdot\| \|$ and a given matrix $A \in \mathbb{R}^{n \times n}$ we may consider the initial growth rate of $A$ with respect to $\|\cdot\| \|$, which can be defined, cf. [7, Chap. 5], as the smallest number $\mu$ so that

$$
\left\|e^{A t}\right\| \leq e^{\mu t}, \quad \text { for all } t \geq 0 .
$$

As the initial growth rate is a finite, convex function and so, in particular, a continuous function of $A$ by [7, Lemma 5.5.9 (vi)] and the set of matrices

$$
\mathcal{M}_{A}^{\text {jump }}:=\left\{A \in \mathbb{R}^{n \times n} \mid \exists \Pi:(A, \Pi) \in \mathcal{M}^{\text {jump }}\right\}
$$

is bounded it follows that there is a constant $C>0$ such that

$$
\left\|e^{A t}\right\| \leq e^{C t}, \quad \text { for all } t \geq 0 \text { and all } A \in \mathcal{M}_{A}^{\text {jump }} .
$$

With this constant and using submultiplicativity it follows that for any element of $\mathcal{S}_{t}$ we have

$$
\begin{aligned}
\left\|\prod_{j=1}^{k} e^{A_{j}^{\mathrm{diff}} \tau_{j}} \Pi_{j}\right\| \leq \prod_{j=1}^{k}\left\|e^{A_{j}^{\mathrm{diff}} \tau_{j}}\right\|\left\|\Pi_{j}\right\| \\
\leq e^{C \sum_{j=1}^{k} \tau_{j}}=e^{C t} .
\end{aligned}
$$

This shows that $\mathcal{S}_{t}$ is bounded for all $t \geq 0$.

(ii) $\Rightarrow$ (i): Fix a $t^{*}>0$ and assume, that $\mathcal{M}_{\Pi}$ is not product bounded. Then we may choose for every constant $C>0$ a $k \in \mathbb{N}$ and $\Pi_{1}, \ldots, \Pi_{k} \in \mathcal{M}_{\Pi}$ such that

$$
\left\|\Pi_{k} \ldots \Pi_{1}\right\|>2 C .
$$

As the map

$$
t \mapsto e^{A_{k} t} \Pi_{k} \ldots e^{A_{1} t} \Pi_{1}
$$

is continuous, there exists an $\varepsilon>0$ such that $\left\|e^{A_{k} t} \Pi_{k} \ldots e^{A_{1} t} \Pi_{1}\right\|>C$ for all $t \in[0, \varepsilon)$. Choose $\tau<\varepsilon$ so that $k \tau<t^{*}$. Then, due to the properties of $\mathcal{M}^{\text {jump }}$,

$$
e^{A_{k}\left(t^{*}-k \tau\right)} e^{A_{k} \tau} \Pi_{k} e^{A_{k-1} \tau} \Pi_{k-1} \ldots e^{A_{1} \tau} \Pi_{1} \in \mathcal{S}_{t^{*}}
$$

and using again submultiplicativity

$$
\begin{aligned}
\left\|e^{-A_{k}\left(t^{*}-k \tau\right)}\right\|\left\|e^{A_{k}\left(t^{*}-k \tau\right)} e^{A_{k} \tau} \Pi_{k} \ldots e^{A_{1} \tau} \Pi_{1}\right\| \\
\geq\left\|e^{A_{k} \tau} \Pi_{k} e^{A_{k-1} \tau} \Pi_{k-1} \ldots e^{A_{1} \tau} \Pi_{1}\right\|>C .
\end{aligned}
$$

Due to compactness of $\mathcal{M}_{A}^{\text {jump }}$ it follows that $\left\|e^{-A_{k}\left(t^{*}-k \tau\right)}\right\|$ is bounded by

$$
\sup \left\{\left\|e^{-A t}\right\| \mid t \in\left[0, t^{*}\right], A \in \mathcal{M}_{A}^{\mathrm{jump}}\right\}<\infty,
$$

which is independent of the choice of $C$ and $k$. Hence unboundedness of $\mathcal{S}_{t^{*}}$ is shown.

We are now able to define the limit growth rate as follows.

Lemma 11 (Boundedness of Lyapunov exponent):

Consider a bounded set $\mathcal{M}^{\text {jump }}$ and assume $\mathcal{M}_{\Pi}^{\text {jump }}$ is product bounded and contains at least one non-zero projector. Then the (upper) Lyapunov exponent given by (11) is well defined and finite.

Proof: Throughout the proof we use the abbreviation $\lambda_{t}$ for $\lambda_{t}\left(\mathcal{S}_{t}\right)$. Due to Lemma 6 and submultiplicativity of induced matrix norms,

$$
\forall \alpha, \beta>0:(\alpha+\beta) \lambda_{\alpha+\beta} \leq \alpha \lambda_{\alpha}+\beta \lambda_{\beta} .
$$

Applying (15) to $\alpha=t-k_{t} \tau$ and $\beta=k_{t} \tau$ for $t>\tau>0$, where $k_{t} \in \mathbb{N}$ is such that $\tau k_{t}<t<\tau\left(k_{t}+1\right)$ and invoking (14) we obtain

$$
\lambda_{t} \leq \varepsilon_{t}+\frac{\tau k_{t}}{t} \lambda_{\tau}
$$

where $\varepsilon_{t} \rightarrow 0$ and $\frac{k_{t} \tau}{t} \rightarrow 1$ as $t \rightarrow \infty$. Hence,

$$
\limsup _{t \rightarrow \infty} \lambda_{t} \leq \inf _{\tau>0} \lambda_{\tau} \leq \liminf _{t \rightarrow \infty} \lambda_{t}
$$

which shows that the limit in the definition of $\lambda(\mathcal{S})$ exists. Furthermore, Proposition 10 yields

$$
\lambda(\mathcal{S})=\inf _{t>0} \lambda_{t}\left(\mathcal{S}_{t}\right)<\infty,
$$

and it remains to show that $\lambda(\mathcal{S}) \neq-\infty$.

Since $\mathcal{M}_{\Pi}^{\text {jump }}$ contains a nontrivial projector, we may consider a nontrivial solution $x(t)=e^{A t} \Pi x^{0}$ with $\Pi x^{0}=x^{0}$ and $(A, \Pi) \in \mathcal{M}^{\text {jump }}$. Then there exists some $\underline{\lambda} \in \mathbb{R}$ such that

$$
\left\|e^{A t} \Pi x^{0}\right\| \geq e^{\underline{\lambda} t}\left\|x^{0}\right\|, \quad \forall t>0 .
$$

Hence $\lambda_{t}\left(\mathcal{S}_{t}\right) \geq \underline{\lambda}$ for all $t>0$ and, therefore, $\lambda(\mathcal{S}) \geq \underline{\lambda}>$ $-\infty$.

Note that $\lambda(\mathcal{S})$ does not depend on the norm chosen to define $\lambda_{t}\left(\mathcal{S}_{t}\right)$. Finally, by choosing the specific sequence $t=$ $1,2,3, \ldots$ for the limit of $\lambda_{t}\left(\mathcal{S}_{t}\right)$ we obtain the following corollary.

Remark 12 (Generalized spectral radius): Consider the discretization of the switched ODE (7) with jumps given by

$$
x(k+1) \in\left\{\Phi_{1} x(k) \mid \Phi_{1} \in \mathcal{S}_{1}\right\}
$$

For this discrete inclusion we can define the generalized spectral radius [19] as usual:

$$
\rho\left(\mathcal{S}_{1}\right):=\lim _{k \rightarrow \infty} \sup _{A_{i} \in \mathcal{S}_{1}}\left\|\prod_{i=1}^{k} A_{i}\right\|^{1 / k} .
$$

Then

$$
\lambda(\mathcal{S})=\ln \rho\left(\mathcal{S}_{1}\right) .
$$

\section{B. Converse Lyapunov Theorem}

We will now show that we can construct a "Lyapunov norm" for the switched ODE with jumps (7) with exponential growth rate arbitrarily close to the upper Lyapunov exponent.

Theorem 13 (Lyapunov norm): Consider the switched ODE with jumps (7) and assume the corresponding Lyapunov exponent $\lambda(\mathcal{S})$ is finite. Then

$$
\|x\|_{\varepsilon}:=\sup _{t>0} \sup _{\Phi_{t} \in \mathcal{S}_{t}} e^{-(\lambda(\mathcal{S})+\varepsilon) t}\left\|\Phi_{t} x\right\|
$$


defines a semi norm which is positive definite on $\operatorname{im} \mathcal{S}$ and satisfies

$$
\left\|\Phi_{t} x\right\|_{\varepsilon} \leq e^{(\lambda(\mathcal{S})+\varepsilon) t}\|x\|_{\varepsilon} .
$$

Proof: $\quad$ Since $\sup _{\Phi_{t} \in \mathcal{S}_{t}}\left\|\Phi_{t} x\right\| \leq e^{\lambda_{t}\left(\mathcal{S}_{t}\right) t}\|x\|$ and $\lambda_{t}\left(\mathcal{S}_{t}\right) \rightarrow \lambda(S)$ as $t \rightarrow \infty$ it follows that

$$
e^{-(\lambda(\mathcal{S})+\varepsilon) t} \sup _{\Phi_{t} \in \mathcal{S}_{t}}\left\|\Phi_{t} x\right\| \rightarrow 0 \quad \text { as } t \rightarrow \infty,
$$

hence there exists $T>0$ such that

$$
\begin{aligned}
\sup _{t>0} \sup _{\Phi_{t} \in \mathcal{S}_{t}} e^{-(\lambda(\mathcal{S})+\varepsilon) t}\left\|\Phi_{t} x\right\| & \\
& =\max _{t \in[0, T]} \sup _{\Phi_{t} \in \mathcal{S}_{t}} e^{-(\lambda(\mathcal{S})+\varepsilon) t}\left\|\Phi_{t} x\right\|
\end{aligned}
$$

which shows that $\|\cdot\|_{\varepsilon}$ is well defined. Furthermore, it is easily seen that $\|x+y\|_{\varepsilon} \leq\|x\|_{\varepsilon}+\|y\|_{\varepsilon}$ and $\|\lambda x\|_{\varepsilon}=$ $|\lambda|\|x\|_{\varepsilon}$ for all $x, y \in \mathbb{R}^{n}, \lambda \in \mathbb{R}$. To show positive definiteness on $\operatorname{im} \mathcal{S}$ let $x \in \operatorname{im} \mathcal{S} \backslash\{0\}$, i.e. there exists $t>0, \Phi_{t} \in \mathcal{S}_{t}$ and $x_{0} \in \mathbb{R}^{n}$ such that $x=\Phi_{t} x_{0}$. As $x \neq 0$ there exists $(A, \Pi) \in \mathcal{M}^{\text {jump }}$ such that $\Pi x=x \neq 0$ and hence $e^{A t} \Pi x \neq 0$ for all $t \geq 0$. This shows that $\|x\|_{\varepsilon}>0$.

To show (16) observe that using the definition

$$
\begin{aligned}
\left\|\Phi_{t} x\right\|_{\varepsilon} & =\sup _{\tau>0 \Phi_{\tau} \in \mathcal{S}_{\tau}} \sup ^{-(\lambda(\mathcal{S})+\varepsilon) \tau}\left\|\Phi_{\tau} \Phi_{t} x\right\| \\
& \leq e^{(\lambda(\mathcal{S})+\varepsilon) t}\|x\|_{\varepsilon} .
\end{aligned}
$$

An immediate consequence of this result is the following converse Lyapunov Theorem.

Corollary 14 (Converse Lyapunov Theorem): Assume that the switched ODE with jumps (7) is (uniformly) exponentially stable, i.e. there exists $\lambda>0$ and $M \geq 1$ such that for all switching signals $\sigma \in \Sigma$ and all corresponding solutions $x$ it holds that

$$
\|x(t)\| \leq M e^{-\lambda t}\|x(0)\| \quad \forall t \geq 0 .
$$

Then $\lambda(\mathcal{S})<0$ and (7) permits a (in general nonsmooth) Lyapunov function $V: \mathbb{R}^{n} \rightarrow \mathbb{R}_{\geq 0}$ given by $V=\|\cdot\|_{\varepsilon}$ as in Theorem 13 with sufficiently small $\varepsilon>0$.

Since trajectories of (7) have jumps it is not clear whether the smoothing technique from [12], [3] can be applied to obtain a smooth Lyapunov function. In particular, the important property

$$
V(\Pi x) \leq V(x) \quad \forall x \in \mathbb{R}^{n}, \Pi \in \mathcal{M}_{\Pi}^{\mathrm{jump}}
$$

might be lost in a convolution of $V$ with a smooth kernel.

\section{Construction of a Barabanov norm}

Even in the case that the Lyapunov norm $\|\cdot\|_{\varepsilon}$ is well defined for $\varepsilon=0$ it would not be an "extremal" norm because in general equality in (16) will not hold for all $x \in \mathbb{R}^{n}$. We are therefore interested in conditions which ensure the existence of a Barabanov norm, defined as follows.

Definition 15 (Barabanov norm): Consider $\mathcal{M}^{\text {jump }} \subseteq \mathcal{A} \Pi$ with corresponding switched ODE with jumps (7) and flows $\mathcal{S}_{t}, t>0$. A norm $\|\cdot\| \|$ is called a Barabanov norm for (7), if there exists a $\lambda \in \mathbb{R}$ such that (i) for all $t \geq 0,(A, \Pi) \in \mathcal{M}^{\text {jump }}, x \in \mathbb{R}^{n}$

$$
\left\|e^{A t} \Pi x\right\| \leq e^{\lambda t}\|x\|
$$

(ii) for all $t>0, x \in \mathbb{R}^{n}$ there exists a $\overline{\Phi_{t}} \in \overline{\mathcal{S}_{t}}$ such that

$$
\left\|\overline{\Phi_{t}} x\right\|=e^{\lambda t}\|x\|,
$$

where $\overline{\mathcal{S}_{t}}$ denotes the closure of $\mathcal{S}_{t}$.

The value $\lambda$ is called exponential growth rate w.r.t. $\|\cdot\| \cdot$.

Note that (17) for $t=0$ implies

$$
\|\Pi x\| \leq\|x\| \quad \forall \Pi \in \mathcal{M}_{\Pi}^{\mathrm{jump}}, x \in \mathbb{R}^{n} .
$$

Furthermore, if (7) admits a Barabanov norm with exponential growth rate $\lambda \in \mathbb{R}$ then (17) and the solution formula analogous to (6) imply for all solutions $x(\cdot)$

$$
\|x(t)\| \leq e^{\lambda t}\left\|x\left(t_{0}-\right)\right\|
$$

and (18) implies that no smaller value fulfills this property.

We will now present our second main result concerning the existence of a Barabanov norm. Recall that a semigroup $\mathcal{S} \subseteq \mathbb{R}^{n \times n}$ is called irreducible, if there are no trivial $\mathcal{S}$ invariant linear subspaces $X \subseteq \mathbb{R}^{n}$. I.e., if $\Phi X \subseteq X$ for all $\Phi \in \mathcal{S}$ then $X=\{0\}$ or $X=\mathbb{R}^{n}$.

Theorem 16 (Barabanov norm): Consider a bounded set

$$
\mathcal{M}^{\text {jump }} \subseteq \mathcal{A} \Pi
$$

and the associated switched ODE with jumps (7). Assume that the semigroup $\mathcal{S}$ defined by (8) and (9) is irreducible. Then the following statements are equivalent:

(i) The set $\mathcal{M}_{\Pi}^{\text {jump }}$ is product bounded.

(ii) The Lyapunov exponent $\lambda(\mathcal{S})$ exists and is finite.

(iii) The switched ODE with jumps (7) admits a Barabanov norm.

Furthermore, in case of existence of a Barabanov norm its exponential growth rate is given by $\lambda(\mathcal{S})$.

Proof: The equivalence of (i) and (ii) is a direct consequence of Proposition 10 and Lemma 11. Furthermore, the existence of a Barabanov norm implies that each flow set $\mathcal{S}_{t}$ is bounded, hence Proposition 10 also shows validity of the implication (iii) $\Rightarrow$ (i). It remains to show existence of a Barabanov norm if the Lyapunov exponent $\lambda(\mathcal{S})$ exists and is finite (note that due to irreducibility Lemma 11 is applicable and so $\lambda(\mathcal{S})>-\infty)$.

As in [19] we define the limit semigroup corresponding to $\mathcal{S}$ by

$$
\mathcal{S}_{\infty}:=\bigcap_{T \geq 0} \overline{\bigcup_{t \geq T} e^{-\lambda(\mathcal{S}) t} \mathcal{S}_{t}}
$$

We note the following properties of $\mathcal{S}_{\infty}$. The proof is a minor modification of that in [19, Prop. 3.2] and therefore omitted.

(i) $\mathcal{S}_{\infty}$ is a compact, irreducible semigroup,

(ii) for all $t>0, \Phi_{t} \in \mathcal{S}_{t}, R \in \mathcal{S}_{\infty}$ we have

$$
e^{-\lambda(\mathcal{S}) t} R \Phi_{t} \in \mathcal{S}_{\infty},
$$

(iii) for all $\Pi \in \mathcal{M}_{\Pi}^{\mathrm{jump}}, R \in \mathcal{S}_{\infty}$ we have

$$
R \Pi \in \mathcal{S}_{\infty},
$$


(iv) for all $t>0, R \in \mathcal{S}_{\infty}$ there exist $\overline{\Phi_{t}} \in \overline{\mathcal{S}_{t}}, \tilde{R} \in \mathcal{S}_{\infty}$ such that

$$
R=e^{-\lambda(\mathcal{S}) t} \overline{\Phi_{t}} \tilde{R} .
$$

We now claim that the norm defined by

$$
\|x\|:=\max \left\{\|S x\| \mid S \in \mathcal{S}_{\infty}\right\}
$$

is a Barabanov norm. Note that $\|\cdot\| \|$ is positive definite by irreducibility of $\mathcal{S}_{\infty}$. Positive homogeneity is clear and $\|\cdot\| \|$ is convex as the maximum of convex functions. This shows that $\|\cdot\|$ is indeed a norm. To show that (17) holds, let $x \in \mathbb{R}^{n}$ and $\Phi_{t}=e^{A t} \Pi \in \mathcal{S}_{t}, t>0$ and $(A, \Pi) \in \mathcal{M}^{\text {jump }}$, then, by (19)

$$
\begin{aligned}
\left\|\Phi_{t} x\right\| & =e^{\lambda(\mathcal{S}) t} \max \left\{\left\|e^{-\lambda(\mathcal{S}) t} R \Phi_{t} x\right\| \mid R \in \mathcal{S}_{\infty}\right\} \\
& \leq e^{\lambda(\mathcal{S}) t} \max \left\{\|S x\| \mid S \in \mathcal{S}_{\infty}\right\}=e^{\lambda(\mathcal{S}) t}\|x\|,
\end{aligned}
$$

which shows (17) for $t>0$. For $t=0$ observe that by (20)

$$
\begin{aligned}
& \|\Pi x\|=\max \left\{\|R \Pi x\| \mid R \in \mathcal{S}_{\infty}\right\} \\
& \quad \leq \max \left\{\|S x\| \mid S \in \mathcal{S}_{\infty}\right\}=\|x\| .
\end{aligned}
$$

Finally, for $x \in \mathbb{R}^{n}$ choose $R \in \mathcal{S}_{\infty}$ such that $\|x\|=\|R x\|$. By (21) we may choose, for any $t>0$, matrices $\tilde{R} \in \mathcal{S}_{\infty}$ and $\overline{\Phi_{t}} \in \overline{\mathcal{S}_{t}}$ such that $R=e^{-\lambda(\mathcal{S}) t} \overline{\Phi_{t}} \tilde{R}$, hence

$$
\begin{aligned}
\left\|\overline{\Phi_{t}} x\right\| \geq e^{\lambda(\mathcal{S}) t}\left\|e^{-\lambda(\mathcal{S}) t} \tilde{R} \overline{\Phi_{t}} x\right\| \\
=e^{\lambda(\mathcal{S}) t}\|R x\|=e^{\lambda(\mathcal{S}) t}\|x\|
\end{aligned}
$$

and the second property (18) is shown.

Remark 17 (Barabanov norm on a subspace): In case that the consistency spaces are restricted to a lower dimensional space $X \subseteq \mathbb{R}^{n}$, i.e., im $\Phi \subseteq X$ for all $\Phi \in \mathcal{S}$, then if $\mathcal{S}$ is irreducible on $X$ the above result yields a Barabanov norm on $X$.

\section{CONCLUSIONS FOR SWITCHED DAES}

We return now to our original problem of switched DAEs

$$
E_{\sigma} \dot{x}=A_{\sigma} x .
$$

We can apply the results from the previous section to obtain the following results about switched DAEs.

Corollary 18 (Converse Lyapunov Theorem): Consider the switched DAE (1) with regular matrix pairs $\left(E_{i}, A_{i}\right)$, $i \in \mathcal{I}$, and switching signals $\sigma \in \Sigma$. Assume that $\mathcal{M}^{\text {jump }}$ as in Theorem 5 is bounded. If (1) is uniformly exponentially stable for all switching signals then there exists a Lyapunov function $V: \mathbb{R}^{n} \rightarrow \mathbb{R}_{\geq 0}$ for (1), in particular, for all consistency projectors $\Pi$,

$$
\forall x \in \mathbb{R}^{n}: V(\Pi x) \leq V(x) .
$$

Proof: Exponential stability implies impulse freeness by definition (cf. [17, Defn. 6.6.1]), which in turn implies index one for all matrix pairs, hence we can apply Theorem 5 to rewrite the switched DAE (1) as a switched ODE with jumps. In particular, uniform exponential stability of (1) implies the same for the corresponding switched ODE with jumps (2). So Corollary 14 yields the converse Lyapunov theorem for switched DAEs.
Corollary 19 (Barabanov norm): Consider the switched DAE (1) with regular index one matrix pairs $\left(E_{i}, A_{i}\right), i \in \mathcal{I}$ and switching signals $\sigma \in \Sigma$. Assume that $\mathcal{M}^{\text {jump }}$ as in Theorem 5 is bounded and $\mathcal{S}$ as given by (8) and (9) is irreducible. Then the following statements are equivalent:

(i) The set of consistency projectors is product bounded.

(ii) The Lyapunov exponent $\lambda(\mathcal{S})$ is bounded.

(iii) There exists a Barabanov norm.

Proof: Follows from Theorems 13 and 16.

Remark 20 (Boundedness of $\mathcal{M}^{\text {jump}}$ ): In both of the above results we need to assume that $\mathcal{M}^{\text {jump }}$ is bounded. This is true if the index set is finite. However, in the general case it is not clear yet whether compactness of the set $\left\{\left(E_{i}, A_{i}\right) \mid i \in \mathcal{I}\right\}$ implies boundedness of the set $\mathcal{M}^{\text {jump }}$.

\section{REFERENCES}

[1] N. E. Barabanov. Absolute characteristic exponent of a class of linear nonstationary systems of differential equations. Siberian Mathematical Journal, 29(4):521-530, 1988.

[2] T. Berger, A. Ilchmann, and S. Trenn. The quasi-Weierstraß form for regular matrix pencils. Lin. Alg. Appl., 436(10):4052-4069, 2012.

[3] C. Cai, A. R. Teel, and R. Goebel. Smooth Lyapunov functions for hybrid systems. I. Existence is equivalent to robustness. IEEE Trans. Autom. Control, 52(7):1264-1277, 2007.

[4] I. Daubechies and J. C. Lagarias. Sets of matrices all infinite products of which converge. Lin. Alg. Appl., 161:227-263, 1992. Corrigenda, ibid., 327:69-83, 2001.

[5] F. R. Gantmacher. The Theory of Matrices (Vol. I \& II). Chelsea, New York, 1959 .

[6] R. Goebel, R. G. Sanfelice, and A. R. Teel. Hybrid dynamical systems. IEEE Control Systems Magazine, 29(2):28-93, 2009.

[7] D. Hinrichsen and A. J. Pritchard. Mathematical Systems Theory I. Modelling, State Space Analysis, Stability and Robustness, volume 48 of Texts in Applied Mathematics. Springer-Verlag, Berlin, 2005.

[8] V. S. Kozyakin. Algebraic unsolvability of problem of absolute stability of desynchronized systems. Autom. Rem. Control, 51(6):754$759,1990$.

[9] D. Liberzon and S. Trenn. On stability of linear switched differential algebraic equations. In Proc. IEEE 48th Conf. on Decision and Control, pages 2156-2161, December 2009.

[10] D. Liberzon and S. Trenn. Switched nonlinear differential algebraic equations: Solution theory, Lyapunov functions, and stability. Automatica, 48(5):954-963, May 2012.

[11] D. Liberzon, S. Trenn, and F. R. Wirth. Commutativity and asymptotic stability for linear switched DAEs. In Proc. 50th IEEE Conf. Decis. Control and European Control Conference ECC 2011, Orlando, USA, pages 417-422, 2011.

[12] Y. Lin, E. D. Sontag, and Y. Wang. A smooth converse Lyapunov theorem for robust stability. SIAM J. Control Optim., 34(1):124-160, 1996.

[13] W. Q. Liu, Y. Yan, and K. L. Teo. On initial instantaneous jumps of singular systems. IEEE Trans. Autom. Control, 40(9):1650-1655, September 1995

[14] A. Tanwani and S. Trenn. On observability of switched differentialalgebraic equations. In Proc. 49th IEEE Conf. Decis. Control, Atlanta, USA, pages 5656-5661, 2010.

[15] S. Trenn. Distributional differential algebraic equations. $\mathrm{PhD}$ thesis, Institut für Mathematik, Technische Universität Ilmenau, Universitätsverlag Ilmenau, Ilmenau, Germany, 2009.

[16] S. Trenn. Regularity of distributional differential algebraic equations. Math. Control Signals Syst., 21(3):229-264, 2009.

[17] S. Trenn. Switched differential algebraic equations. In F. Vasca and L. Iannelli, editors, Dynamics and Control of Switched Electronic Systems - Advanced Perspectives for Modeling, Simulation and Control of Power Converters, chapter 6, pages 189-216. Springer-Verlag, London, 2012.

[18] K. Weierstraß. Zur Theorie der bilinearen und quadratischen Formen Berl. Monatsb., pages 310-338, 1868.

[19] F. R. Wirth. The generalized spectral radius and extremal norms. Lin. Alg. Appl., 342:17-40, 2002. 